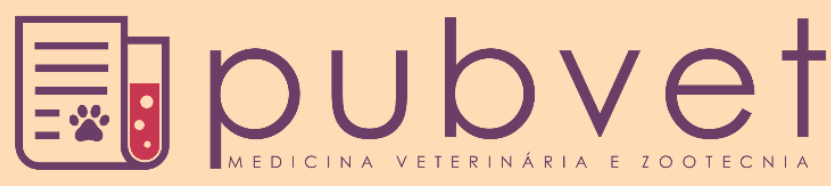

https://doi.org/10.31533/pubvet.v13n10a433.1-9

\title{
Diagnóstico citopatológico de criptococose em gata: Relato de caso
}

\author{
Jéssica Ragazzi Calesso $^{1 * \bullet}{ }^{\bullet}$, Vinicius Prado de Oliveira $^{2} \bullet$, Melissa Gonçalves Jacob ${ }^{2} \bullet$, Ademir \\ Zacarias Junior $^{3} \bullet$, Karina Yukie Hirata ${ }^{4}$ \\ ${ }^{I}$ Médica Veterinária Mestranda em Ciência Animal - Departamento de Clínica e Cirurgia Veterinárias, Universidade Federal de Minas \\ Gerais (UFMG), Belo Horizonte - MG, Brasil \\ ${ }^{2}$ Residente em Medicina Veterinária - Hospital Veterinário Escola, Universidade Estadual do Norte do Paraná (UENP), Bandeirantes - PR, Brasil \\ ${ }^{3}$ Docente do curso de Medicina Veterinária - Universidade Estadual do Norte do Paraná (UENP), Bandeirantes - PR, Brasil \\ ${ }^{4}$ Docente do curso de Medicina Veterinária - Instituto Federal de Minas Gerais (IFMG), Bambuí - MG, Brasil \\ *Autor para correspondência: jessicacalesso@gmail.com
}

Resumo. O objetivo do trabalho foi relatar um caso de criptococose cutânea e nasal em uma gata, adulta, que apresentava neoformação firme em região cefálica, aderida, não alopecia e não ulcerada, a qual provocava assimetria facial, associada a presença discreta de secreção nasal mucoide unilateral. As infecções micóticas podem ser classificadas quanto ao local da lesão em cutâneas, subcutâneas e sistêmicas. A espécie felina é altamente suscetível a diversas infecções fúngicas, dentre elas, a criptococose. A criptococose é cerca de sete a dez vezes mais comuns em gatos do que em cães e os sinais clínicos são variados e inespecíficos, uma vez que pode acometer sistema respiratório, tegumentar, ocular e sistema nervoso central. Seu diagnóstico baseia-se inicialmente nas informações obtidas na anamnese e ao exame físico e pode ser confirmado por diversos métodos, como exame citológico, cultura microbiológica, exame histopatológico ou através de técnicas moleculares. O diagnóstico da paciente deste estudo se firmou por meio de exame citopatológico da neoformação, que revelou a presença de numerosas formas leveduriformes encapsuladas compatíveis com microrganismos do gênero Cryptococcus. A terapia instituída com sucesso foi o itraconazol na dose de $10 \mathrm{mg} / \mathrm{kg}$ por via oral a cada 24 horas durante 8 meses, sem ocorrência de recidivas até o momento. Apesar de não ser considerada uma antropozoonose, a criptococose merece atenção na clínica de animais de companhia, pois seu diagnóstico é difícil, e frequentemente se dá apenas por achados post mortem. Além disso, se não tratada eficazmente o prognóstico é desfavorável, podendo levar o paciente a óbito.

Palavras chave: Cryptococcus spp., dermatopatia fúngica, granuloma, micose sistêmica, trato respiratório

\section{Cytopathological diagnosis of cryptococcosis in a cat: Case report}

Abstract. The objective of this study was to report a case of cutaneous and nasal cryptococcosis in an adult female cat who presented firm, non-alopecic and non-ulcerated cephalic neoformation, which caused facial asymmetry, associated with the slight presence of unilateral mucous nasal discharge. Mycotic infections can be classified according to the lesion site in cutaneous, subcutaneous and systemic. The feline species is highly susceptible to several fungal infections, including cryptococcosis. Cryptococcosis is about seven to ten times more common in cats than dogs, and clinical signs are varied and nonspecific, as they may affect the respiratory, integumentary, ocular, and central nervous system. Its diagnosis is based initially on the information obtained from the anamnesis and physical examination and can be confirmed by several methods, such as cytological examination, microbiological culture, histopathological examination or through molecular techniques. The patient's diagnosis in this study was established by cytopathological examination of the neoformation, which revealed the presence of numerous encapsulated yeast forms 
compatible with Cryptococcus microorganisms. The successfully instituted therapy was 10 $\mathrm{mg} / \mathrm{kg}$ oral itraconazole every 24 hours for 8 months, with no recurrences so far. Although not considered an anthropozoonosis, cryptococcosis deserves attention in the small animal clinic because its diagnosis is difficult, and often occurs only by postmortem findings. In addition, if not effectively treated the prognosis is unfavorable and may lead to death.

Keywords: Cryptococcus spp., fungal dermatopathy, granuloma, systemic mycosis, respiratory tract

\section{Diagnóstico citopatológico de la criptococosis en gato: reporte de un caso}

Resumen. El objetivo de este estudio fue informar un caso de criptococosis cutánea y nasal en una gata adulta que presentaba una neoformación cefálica firme, no alopécica y no ulcerada, que causaba asimetría facial, asociada con la leve presencia de secreción nasal mucoide unilateral. Las infecciones micóticas se pueden clasificar según el sitio de la lesión en cutáneas, subcutáneas y sistémicas. La especie felina es altamente susceptible a varias infecciones fúngicas, incluida la criptococosis. La criptococosis es aproximadamente siete a diez veces más común en gatos que en perros, y los signos clínicos son variados e inespecíficos, ya que pueden afectar el sistema respiratorio, tegumentario, ocular y sistema nervioso central. Su diagnóstico se basa inicialmente en la información obtenida de la anamnesis y el examen físico y puede confirmarse mediante varios métodos, como el examen citológico, el cultivo microbiológico, el examen histopatológico o mediante técnicas moleculares. El diagnóstico de la paciente en este estudio se estableció mediante un examen citopatológico de la neoformación, que reveló la presencia de numerosas formas de levadura encapsulada compatibles con microorganismos Cryptococcus. La terapia instituida con éxito fue de $10 \mathrm{mg} / \mathrm{kg}$ de itraconazol vía oral a cada 24 horas durante 8 meses, sin recurrencias hasta el momento. Aunque no se considera una antropozoonosis, la criptococosis merece atención en la clínica de pequeños animales porque su diagnóstico es difícil y a menudo ocurre solo por hallazgos post mortem. Además, si no se trata eficazmente, el pronóstico es desfavorable y puede provocar la muerte.

Palabras clave: Cryptococcus spp., dermatopatía fúngica, granuloma, micosis sistémica, tracto respiratório

\section{Introdução}

A criptococose em animais de companhia é pouco frequente em comparação a outras doenças fúngicas superficiais e subcutâneas (Larsson et al., 2003) e representa a micose sistêmica mais comum na espécie felina (Brito-Santos et al., 2019; Caswell \& Williams, 2007; Miller et al., 2013; Myers et al., 2017). No entanto, de acordo Brito-Santos et al. (2019) desde a primeira descrição no Brasil em 1971, apenas 27 casos de criptococose felina foram relatados. É uma doença fúngica oportunista (Pereira et al., 2013), cosmopolita, que acomete o homem e os animais, causados por leveduras dos complexos Cryptococcus neoformans e Cryptococcus gattii (Kwon-Chung et al., 2017). A transmissão ocorre pela inalação do microrganismo presente em matéria orgânica, como excrementos de pássaros e morcegos, além de eucalipto em decomposição em poeira contaminada (Rondelli et al., 2010). A infecção ocorre no trato respiratório, podendo se disseminar por via hematógena (Castellá et al., 2008; Miller et al., 2013; Pereira et al., 2013) para o encéfalo, olhos, pele e outros órgãos (Queiroz, 2008). Os sinais clínicos são variados e muitas vezes inespecíficos (Castro et al., 2017). Espirros, secreção nasal uni ou bilateral, lesões granulomatosas e deformidade facial são frequentes. Massas cutâneas ou subcutâneas, únicas ou múltiplas também são comumente encontradas em gatos com criptococose (Nelson \& Couto, 2015).

O diagnóstico pode ser confirmado por exame citológico, cultura microbiológica, exame histopatológico ou pelas técnicas moleculares (Hnilica \& Medleau, 2012; Medleau et al., 2003; Trindade et al., 2008). Entretanto, o diagnóstico da criptococose frequentemente se dá apenas por achados post mortem (Rondelli et al., 2010). O tratamento consiste no uso de antifúngicos sistêmicos por um longo período para eliminar a infecção (Miller et al., 2013).

O presente trabalho teve como objetivo relatar um caso de criptococose em uma gata cujo diagnóstico foi obtido através de citologia aspirativa por agulha fina. 


\section{Relato de caso}

Foi atendida no Hospital Veterinário Escola da Universidade Estadual do Norte do Paraná uma gata, adulta, não castrada, $2,5 \mathrm{~kg}$, com histórico de aumento de volume em região cefálica, dispneia, espirros e secreção nasal com evolução de 30 dias. A tutora havia resgatado a paciente há poucos meses e relatou normorexia, normoquezia, normodipsia e normúria, negando alterações gastrointestinais, neurológicas, locomotoras e cardiovasculares. Ao exame físico, os parâmetros fisiológicos (frequência cardíaca e respiratória, temperatura retal, tempo de perfusão capilar e coloração de mucosas) encontravam-se dentro da normalidade para a espécie, entretanto, ambos os linfonodos submandibulares se encontravam aumentados à palpação e a paciente apresentava escore corporal magro. Ao exame físico geral, não foram constatadas alterações à auscultação pulmonar. À avaliação dermatológica foi observada presença de neoformação firme em região cefálica, aderida, não alopécica e não ulcerada, medindo $2 \times 5 \times 1 \mathrm{~cm}$ (Figura 1A), provocando assimetria facial, e presença discreta de secreção nasal mucoide unilateral (Figura 1B).
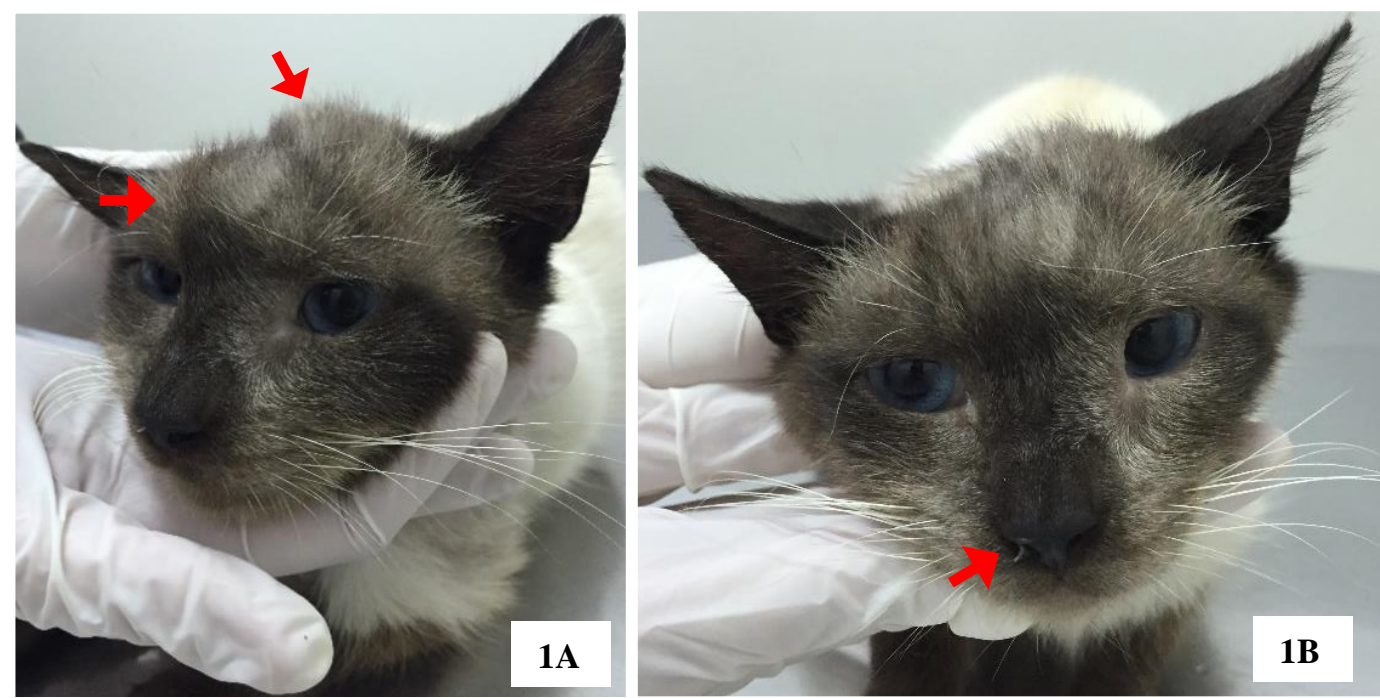

Figura 1. (A): Neoformação firme em região cefálica (setas vermelhas), aderida, não alopécica e não ulcerada, medindo $2 \times 5 \times 1 \mathrm{~cm}$; (B): Assimetria facial, e discreta secreção nasal mucoide unilateral em gata com criptococose.

Foram solicitados exames complementares como hemograma completo e bioquímico sérica de enzimas renais e hepáticas. Ao leucograma foi observada leucocitose por neutrofilia com desvio à esquerda regenerativo (Tabela 1). Os demais resultados apresentaram-se dentro da normalidade para a espécie. Foi realizado exame citopatológico, com coloração de Romanowsky, de material obtido através de citologia aspirativa por agulha fina (CAAF) da neoformação, a qual confirmou o diagnóstico de criptococose (Figura 2). Também foi realizada cultura fúngica, entretanto, apresentou-se negativa. $\mathrm{O}$ diagnóstico de infecção pelo vírus da imunodeficiência felina (FIV) e vírus da leucemia felina (FeLV) foi realizado pelo método de reação em cadeia polimerase (PCR), o qual foi negativo para ambas as enfermidades.

Tabela 1. Leucograma com leucocitose por neutrofilia com desvio à esquerda regenerativo em gata com criptococose.

\begin{tabular}{lll}
\hline Leucograma & Resultados & Valores de Referência \\
\hline Leucócitos totais & $29.500 / \mu \mathrm{L}$ & $5.500-19.500 / \mu \mathrm{L}$ \\
Bastonetes & $885 / \mu \mathrm{L}$ & $0-300 / \mu \mathrm{L}$ \\
Segmentados & $23.010 / \mu \mathrm{L}$ & $2.500-12.500 / \mu \mathrm{L}$ \\
Linfócitos & $5.605 / \mu \mathrm{L}$ & $1.500-7.000 / \mu \mathrm{L}$ \\
\hline
\end{tabular}




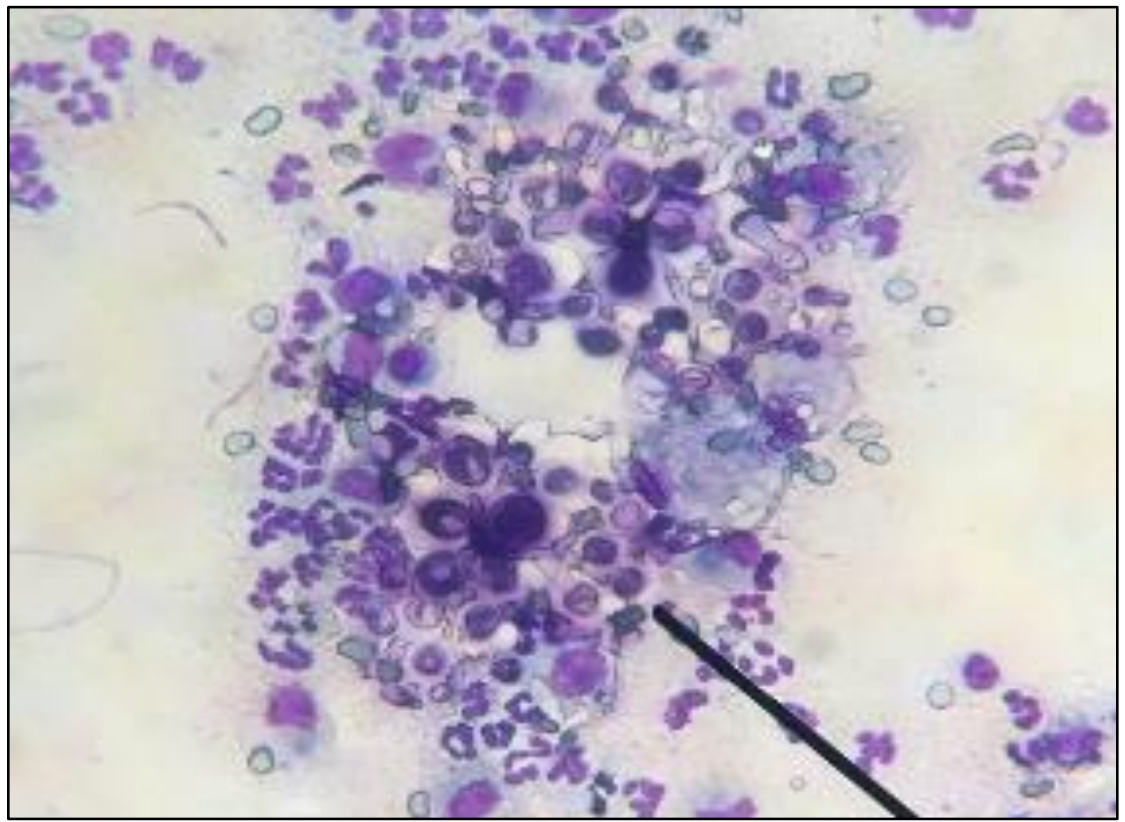

Figura 2. Exame citopatológico de material obtido por citologia aspirativa por agulha fina de neoformação cutânea, com a presença de numerosas formas leveduriformes encapsuladas compatíveis com o gênero Cryptococcus.

Após diagnóstico, foi instituída terapia antifúngica com itraconazol na dose de $10 \mathrm{mg} / \mathrm{kg}$ por via oral a cada 24 horas, até novas recomendações, e antibacteriana com amoxicilina e ácido clavulânico na dose de $15 \mathrm{mg} / \mathrm{kg}$ por via oral a cada 12 horas durante 21 dias. A antibioticoterapia foi prescrita, pois dias após o atendimento ocorreu infecção bacteriana secundária da neoformação cutânea, drenando conteúdo serossanguinolento. Ao primeiro retorno, 3 semanas após início da terapia, a paciente apresentava melhora clínica, em que a tutora relatou redução dos espirros e da secreção nasal, e melhora laboratorial, com leucograma dentro dos valores de normalidade para a espécie. A partir de então, foi mantida apenas terapia antifúngica prolongada por 8 meses, com retornos realizados a cada 2 meses para acompanhamento clínico e laboratorial da paciente. Como inicialmente a paciente encontrava-se com sua nutrição comprometida, pesando $2,5 \mathrm{~kg}$, com o tratamento e melhora de sua nutrição, houve ganho de peso $(3,5 \mathrm{~kg}$,) e ajuste na posologia do itraconazol para manutenção de $10 \mathrm{mg} / \mathrm{kg} / \mathrm{dia}$. A cura clínica foi alcançada ao final do quarto mês de tratamento (Figura 3A) e, ao final da terapia, a paciente encontrava-se em bom estado geral e ausência de manifestações clínicas da criptococose (Figura 3B).

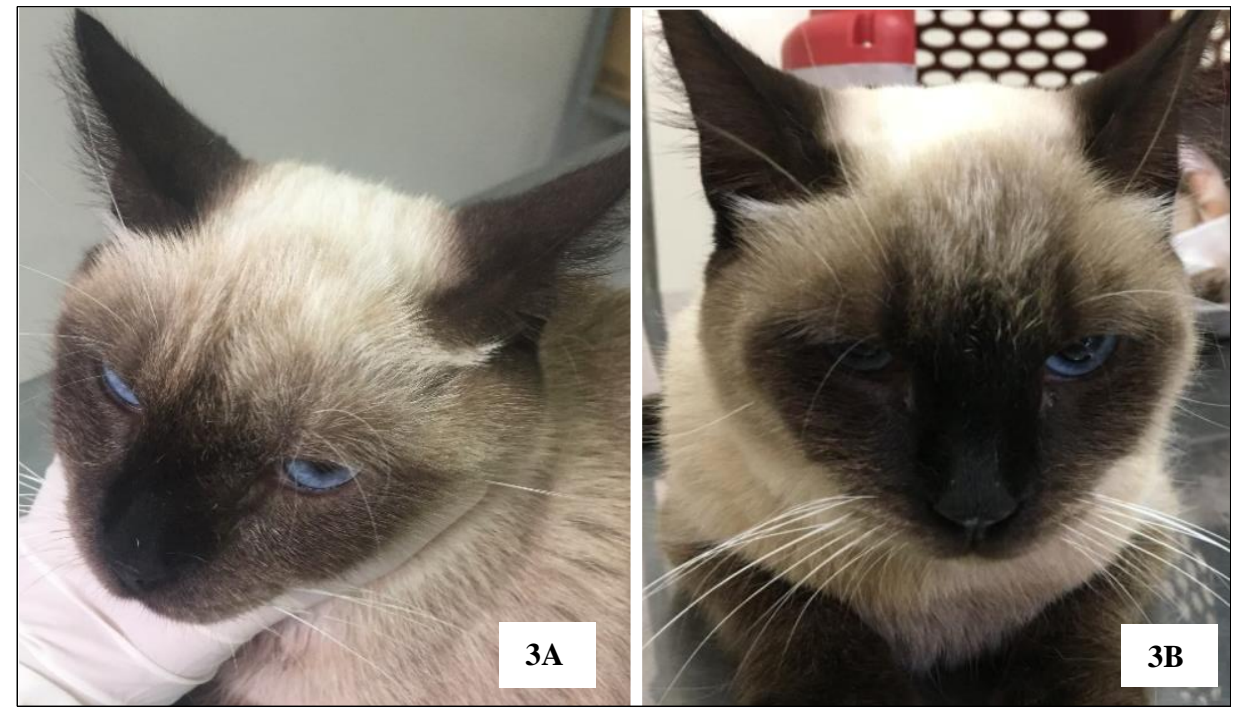

Figura 3. (3A): Gata com criptococose apresentando cura clínica da doença após término do $4^{\circ}$ mês de tratamento com itraconazol; (3B): Gata com criptococose em alta após 8 meses de tratamento com itraconazol e remissão completa da doença. 


\section{Discussão}

As infecções micóticas muitas vezes não apresentam alterações clínicas e lesões macroscópicas características que permitam um diagnóstico presuntivo (Galiza et al., 2014). Portanto, as manifestações clínicas da criptococose dependem amplamente do sistema acometido: respiratório, tegumentar, ocular e sistema nervoso central (SNC), podendo afetar um ou mais dos sistemas supracitados (Corrêa, 1994).

A paciente manifestou quadro respiratório e dermatológico, em que o último foi caracterizado por neoformação em região cefálica. Segundo Kerl (2003), em aproximadamente $40 \%$ dos casos pode haver envolvimento cutâneo e as lesões criptocócicas cutâneas geralmente são representadas por massas dérmicas ou subcutâneas, firmes e bem circunscritas, que podem ulcerar e drenar exsudato seroso ou mucoide (Corrêa, 1994; Gross et al., 2008). As neoformações podem medir desde $2 \mathrm{~mm}$ à $5 \mathrm{~cm}$ de diâmetro, sendo acompanhados ou não de alopecia (Corrêa, 1994), corroborando o presente caso. Raramente, a infecção pode ocorrer na forma de lesões localizadas na pele ou no tecido subcutâneo, decorrentes de inoculação direta do agente através de uma ferida penetrante (Miller et al., 2013).

A síndrome cutânea ocorre mais comumente nos gatos, sendo pouco comum em cães. As lesões estão localizadas principalmente na cabeça e pescoço (Larsson et al., 2003), dados compatíveis com o observado na paciente do presente relato. Isso ocorre devido à inoculação do agente e desenvolvimento de infecção localizada, caracterizada por lesões granulomatosas na cavidade nasal, chamadas popularmente como "nariz de palhaço", uma vez que os seios nasais dos gatos possuem uma filtragem mais eficiente de pequenas partículas (Pereira et al., 2013; Trindade et al., 2008). Apesar de a paciente não apresentar o sinal clínico característico de "nariz de palhaço", a massa encontrava-se em região de cabeça. As lesões ainda podem surgir como erosões e ulcerações, as quais podem ser nasais, linguais, palatinas, gengivais, labiais, podais e no leito ungueal (Larsson et al., 2003). Deformações faciais foram citadas por Corrêa (1994) e Nelson \& Couto (2015), como a assimetria facial observada neste caso.

Em trabalho de Galiza et al. (2014) foram analisados os aspectos clínico-epidemiológicos, histomorfológicos e histoquímicos de sete casos de criptococose diagnosticados em animais de companhia, em que seis eram da espécie felina. Dos seis gatos com a doença confirmada, apenas dois apresentaram sinais clínicos cutâneos. Um apresentando feridas ulceradas em cabeça, pescoço, região periorbital, comissura labial e membros, e outro apresentando lesão em dígito do membro pélvico esquerdo. Já em estudo retrospectivo de doenças micóticas em gatos realizado por Castro et al. (2017), em 13 gatos com criptococose confirmada, oito apresentaram sinais clínicos cutâneos como nódulos, ulcerações e exsudação serossanguinolenta, esta última compatível com o conteúdo do abscesso da paciente relatada.

Os sinais clínicos respiratórios apresentados pela paciente corroboram os encontrados na literatura, os quais incluem espirros, descarga nasal uni ou bilateral serossanguinolenta ou mucopurulenta e dispneia, sendo raro o envolvimento pulmonar (Corrêa, 1994). As alterações neurológicas estão associadas à meningite criptocócica, em que podem ser observados sinais como depressão, anorexia, palidez de mucosas, head pressing, sialorreia, paresia, perda de tônus muscular e coma. As alterações oculares envolvem retinites, coriorretinites granulomatosas, cistos exsudativos, descolamento de retina e uveíte anterior (Corrêa, 1994). A gata em questão não apresentou outras alterações além do quadro respiratório e dermatológico. Queiroz (2008) e Trindade et al. (2008) descreveram que o agente provavelmente se dissemina para os linfonodos regionais através dos vasos linfáticos presentes na cavidade nasal, justificando o aumento de volume nos linfonodos submandibulares. Miller et al. (2013) e Nelson \& Couto (2015) também citam que a linfadenopatia mandibular está frequentemente presente. Quanto às alterações laboratoriais, leucocitose neutrofílica com desvio à esquerda regenerativo também já foi observada por outros autores (Rondelli et al., 2010), os quais também observaram linfopenia e discreta trombocitose. As duas últimas alterações não foram observadas no presente caso. Entretanto, segundo Nelson \& Couto (2015), as anormalidades hematológicas mais comuns são anemia não regenerativa e monocitose.

A dificuldade na formulação de um diagnóstico sugestivo de criptococose pode estar diretamente associada à presença de sinais clínicos inespecíficos, diversidade na sua apresentação clínica e distribuição das lesões (Pereira et al., 2013; Trindade et al., 2008) como observado em outros trabalhos (Castro et al., 2017; Galiza et al., 2014). Em estudo realizado por Galiza et al. (2014), somente em dois de seis gatos houve suspeita clínica de criptococose. Já em estudo conduzido por Castro et al. (2017), a suspeita de doença fúngica esteve presente em quatro de 13 animais. Quando não é possível a cultura 
fúngica, que permite a identificação definitiva do agente, o reconhecimento das características morfológicas das leveduras de Cryptococcus spp. é a principal ferramenta de diagnóstico (Guarner \& Brandt, 2011). De acordo com Medleau et al. (2003) o método mais rápido de diagnóstico é feito por exame citológico de exsudato nasal ou de lesões de pele, fluido cerebroespinhal e aspirado de tecidos, o qual pode revelar a presença de leveduras esféricas encapsuladas. Em Medicina Veterinária, os primeiros trabalhos a utilizar a citologia aspirativa por agulha fina (CAAF) na rotina clínica datam da década de 1980, mostrando sua utilização no diagnóstico de desordens neoplásicas, hiperplásicas, degenerativas e inflamatórias de pequenos animais. É um método simples e de baixo custo, pois não envolve equipamentos sofisticados (Guedes et al., 2000). Nas doenças infecciosas, a CAAF desempenha um papel importante, apresentando riscos menores que a biópsia cirúrgica (Martins et al., 2008), uma vez que o exame citológico pode ter uma acurácia de $83,3 \%$ em relação aos resultados do histopatológico (Guedes et al., 2000).

A técnica de CAAF como método diagnóstico da criptococose em animais de companhia já foi relatada como eficiente por outros autores (Brito-Santos et al., 2019; Castro et al., 2017; Letendre \& Boysen, 2015; Pereira et al., 2013; Souza et al., 2014; Trindade et al., 2008). Um exemplo de sua eficiência foi relatado por Souza et al. (2014), que realizaram a coleta do material de um cão através de uma toracotomia exploratória. Segundo os autores, a abordagem foi essencial para que se chegasse a um diagnóstico, uma vez que exames radiográficos não foram precisos e a cultura foi negativa. Situação semelhante ocorreu com a paciente do presente relato, cuja cultura fúngica de material obtido de massa em região cefálica apresentou-se negativa e o exame citopatológico forneceu um diagnóstico mais rápido e eficiente.

Os achados citopatológicos encontrados nesse caso corroboram aos encontrados em outros trabalhos (Martins et al., 2008; Trindade et al., 2008), os quais revelaram a presença de estruturas leveduriformes, ovais a arredondadas, apresentando cápsula ao seu redor, compatíveis com Cryptococcus spp., com presença predominante de infiltrado neutrofílico, além de macrófagos ativados fagocitando leveduras. Além de que, a espessa cápsula de lipídeo não se cora, formando, assim, um halo claro ou refratário (Miller et al., 2013; Rondelli et al., 2010). Apesar das vantagens do exame citopatológico, a cultura é o exame comprobatório da doença. O Cryptococcus spp. cresce bem em vários meios de cultivos, que não contenham ciclo-heximida, como Ágar-sangue, Ágar-Sabouraud e Ágar infusão de cérebro-coração. O fungo pode crescer em temperaturas entre $25^{\circ} \mathrm{C}$ e $37^{\circ} \mathrm{C}$, mas cresce melhor a $30^{\circ} \mathrm{C}$, sendo a termotolerância máxima de $40^{\circ} \mathrm{C}$. Colônias mucóides de tonalidade creme podem ser observadas em 48 horas de incubação (Kon et al., 2008).

A criptococose engloba dois tipos de infecções, considerando aspectos clínicos e epidemiológicos: uma causada pelo Cryptococcus neoformans, de caráter oportunista, cosmopolita, associada à imunossupressão celular e outra causada pelo Cryptococcus gattii, uma infecção primária, de hospedeiro imunocompetente, endêmica em áreas tropicais e subtropicais (Kon et al., 2008). Quatro sorotipos são descritos, sendo os sorotipos A e D pertencentes à Cryptococcus neoformans var. grubii e Cryptococcus neoformans var. neoformans, respectivamente, e os sorotipos $\mathrm{B}$ e C pertencentes a Cryptococcus gattii (Bovers et al., 2008; Miller et al., 2013). Os sorotipos A e D são evidenciados em fezes de pombos e estão associados às infecções criptocócicas em felinos brasileiros (Pereira et al., 2013). Além disso, o sorotipo D também está presente em plantações de eucaliptos em climas tropicais, sendo esta mais uma fonte de infecção potencial para a doença (Caswell \& Williams, 2007; Ribeiro et al., 2006). Como no caso em questão a cultura fúngica foi negativa, não foi possível a determinação de qual agente era causador da infecção na paciente. Entretanto, suspeitou-se de infecção por Cryptococcus neoformans por seus aspectos clínicos e epidemiológicos citados anteriormente. Segundo a literatura, a criptococose felina está associada principalmente às infecções virais pelos vírus da imunodeficiência felina e leucemia felina (FIV e FeLV, respectivamente) devido imunossupressão (Miller et al., 2013; Nelson \& Couto, 2015). Apesar de a paciente ter exames negativos para FIV e FeLV, presume-se que estava em condição de imunossupressão por ser um animal errante e em má nutrição.

O exame histopatológico é realizado baseando-se na morfologia específica do agente e em suas características histoquímicas típicas, principalmente naqueles casos em que a cultura micológica não está disponível. Para isso é necessário o conhecimento do padrão morfológico e histoquímico da infecção por Cryptococcus spp., assim como suas variações nos tecidos afetados (Galiza et al., 2014). Como o diagnóstico da paciente foi alcançado em exame citopatológico e apresentou boa resposta 
terapêutica, o exame histopatológico não foi realizado. Testes de ELISA detectam tanto antígenos como anticorpos, e detectam antígenos em títulos mais baixos e mais precocemente na infecção criptocócica. No entanto, é um exame mais demorado e laborioso (Kon et al., 2008). Embora não seja a técnica mais empregada na rotina clínica, a reação em cadeia pela polimerase (PCR) tem sido utilizada com bons resultados em centros de pesquisa.

O tratamento para criptococose consiste no uso de antifúngicos sistêmicos, os quais devem ser administrados por um longo período para garantir que a infecção seja eliminada. Isso pode exigir até dois anos de tratamento (Miller et al., 2013) e se estender a terapia por um a dois meses após a resolução dos sinais clínicos (Nelson \& Couto, 2015). O tratamento da paciente foi suspenso após exame citopatológico negativo, conforme descrito por Miller et al. (2013) e o período total de tratamento de oito meses corrobora outros trabalhos (Larsson et al., 2003; Letendre \& Boysen, 2015). As medicações recomendadas são anfotericina B, flucitosina, cetoconazol, itraconazol, fluconazol, isoladamente ou em associações (Larsson et al., 2003). As drogas antifúngicas azólicas são os fármacos de escolha para animais menos afetados (Miller et al., 2013) e, por isso, a droga utilizada para o tratamento da gata do presente trabalho foi o itraconazol, que tem um índice terapêutico favorável e é considerado de escolha para o tratamento da maioria dos casos de criptococose em gatos (Miller et al., 2013). O cetoconazol é o mais barato dos medicamentos disponíveis e tem sido eficaz em alguns pacientes, apesar dos efeitos colaterais gastrointestinais (Miller et al., 2013). O fluconazol tem boa eficácia, mas a resistência in vivo já foi relatada por Sykes et al. (2017).

As infecções fúngicas, quando não tratadas eficazmente, associadas à má nutrição corroboram para um prognóstico desfavorável, podendo levar o paciente a óbito (Trindade et al., 2008). A paciente apresentava um bom prognóstico, uma vez que não apresentava infecção por retroviroses e, apesar da condição de má nutrição observada na primeira consulta, respondeu de forma excelente ao tratamento com o itraconazol, sem intercorrências durante a terapia.

\section{Conclusões}

A criptococose em animais de companhia é considerada pouco frequente em comparação a outras doenças fúngicas superficiais e subcutâneas e, apesar de a espécie felina ser mais suscetível que a canina para infecções por Cryptococcus spp., o diagnóstico presuntivo é considerado difícil pela variedade de sistemas acometidos e manifestações clínicas. Por isso, muitas vezes o diagnóstico da criptococose se dá apenas por achados post mortem. Com o presente relato foi possível observar que o exame citopatológico é um método simples, de baixo custo, com menores riscos para o paciente, com boa acurácia e que permite um diagnóstico mais rápido da doença para instituição da terapia, visando melhor prognóstico para o animal.

\section{Referências bibliográficas}

Bovers, M., Hagen, F. \& Boekhout, T. (2008). Diversity of the Cryptococcus neoformans-Cryptococcus gattii species complex. Revista Iberoamericana de Micologia, 25(1):4-12.

Brito-Santos, F., Reis, R. S., Coelho, R. A., Almeida-Paes, R., Pereira, S. A., Trilles, L., . . Gremião, I. D. F. (2019). Cryptococcosis due to Cryptococcus gattii VGII in southeast Brazil: The one health approach revealing a possible role for domestic cats. Medical Mycology Case Reports, 2461-64.

Castellá, G., Abarca, M. L. \& Cabañes, F. J. (2008). Criptococosis y animales de compañía. Revista Iberoamericana de Micología, 25(1):S19-S24.

Castro, N. B., Rolim, V. M., Nascimento, L. C., Silveira, A. F. V., Argenta, F. F., Ferreiro, L., . . Sonne, L. (2017). Doenças micóticas em gatos no Rio Grande do Sul. Pesquisa Veterinária Brasileira, 37(11):1313-1321.

Caswell, J. L. \& Williams, K. J. (2007). Infectious diseases of the respiratory system. Jubb, Kennedy, and Palmer's Pathology of Domestic Animals, 2635-653.

Corrêa, G. L. B. (1994). Criptococose em gatos. Ciência Rural, 24(2):431-437.

Galiza, G. J. N., Silva, T. M., Caprioli, R. A., Tochetto, C., Rosa, F. B., Fighera, R. A. \& Kommers, G. D. (2014). Características histomorfológicas e histoquímicas determinantes no diagnóstico da criptococose em animais de companhia. Pesquisa Veterinária Brasileira, 34(3):261-269. 
Gross, T. L., Ihrke, P. J., Walder, E. J. \& Affolter, V. K. (2008). Skin diseases of the dog and cat: clinical and histopathologic diagnosis. Oxford: John Wiley \& Sons.

Guarner, J. \& Brandt, M. E. (2011). Histopathologic diagnosis of fungal infections in the 21st century. Clinical Microbiology Reviews, 24(2):247-280.

Guedes, R. M. C., Zica, K. G. B., Coelho-Guedes, M. I. M. \& Oliveira, S. R. (2000). Acurácia do exame citológico no diagnóstico de processos inflamatórios e proliferativos dos animais domésticos. Arquivo Brasileiro de Medicina Veterinária e Zootecnia, 52(5):437-439.

Hnilica, K. A. \& Medleau, L. (2012). Dermatologia de pequenos animais: atlas colorido e guia terapêutico. Rio de Janeiro, Rio de Janeiro, Brasil. : Roca.

Kerl, M. E. (2003). Update on canine and feline fungal diseases. The Veterinary Clinics of North America. Small Animal Practice, 33(4):721-747.

Kon, A. S., Grumach, A. S., Colombo, A. L., Penalva, A. C. O., Wanke, B., Telles, F. Q., . . Ponzio, V. (2008). Consenso em criptococose-2008. Revista da Sociedade Brasileira de Medicina Tropical, 41(5):524-544.

Kwon-Chung, K. J., Bennett, J. E., Wickes, B. L., Meyer, W., Cuomo, C. A., Wollenburg, K. R., . . Chen, J. (2017). The case for adopting the "species complex" nomenclature for the etiologic agents of cryptococcosis. MSphere, Clinical Science and Epidemiology, 2(1):e00357-00316.

Larsson, C. E., Otsuka, M., Michalany, N. S., Barros, P. S. M., Gambale, W. \& Safatle, A. M. V. (2003). Criptococose canina: relato de caso. Arquivo Brasileiro de Medicina Veterinária e Zootecnia, 55(5):533-538.

Letendre, J.-A. \& Boysen, S. (2015). Cranial vena cava syndrome secondary to cryptococcal mediastinal granuloma in a cat. The Canadian Veterinary Journal, 56(4):365-369.

Martins, D. B., Barbosa, A. L. T., Cavalheiro, A., Lopes, S. T. d. A., Santurio, J. M., Schossler, J. E. \& Mazzanti, A. (2008). Diagnosis of canine cryptococcosis by fine-needle aspiration cytology. Ciência Rural, 38(3):826-829.

Medleau, L., Hnilica, K. A. \& Fagliari, G. S. (2003). Dermatologia de pequenos animais: atlas colorido e guia terapêutico. São Paulo: Roca.

Miller, W. H., Griffin, C. E., Campbell, K. L. \& Muller, G. H. (2013). Muller and Kirk's Small Animal Dermatology. Philadelphia, USA: Elsevier Health Sciences.

Myers, A., Meason-Smith, C., Mansell, J., Krockenberger, M., Peters-Kennedy, J., Payne, H. R. \& Hoffmann, A. R. (2017). Atypical cutaneous cryptococcosis in four cats in the USA. Veterinary Dermatology, 28(4):405-e497.

Nelson, R. W. \& Couto, C. G. (2015). Medicina interna de pequenos animais. Amsterdan: Elsevier Editora.

Pereira, M. F., Santos, B. M., Silva, V. C. L., Pereira, H. N. S., Silva, L. B. G., Paiva, B. H. A., . . Oliveira, A. A. F. (2013). Aspectos clínicos e anatomopatológicos da criptococose nasal com disseminação sistêmica em cão: relato de caso. Medicina Veterinária, 7(2):7-15.

Queiroz, J. P. A. F. (2008). Criptococose-Uma revisão bibliográfica. Acta Veterinaria Brasilica, 2(2):32-38.

Ribeiro, Â. M., Silva, L. K. R. E., Silveira Schrank, I., Schrank, A., Meyer, W. \& Henning Vainstein, M. (2006). Isolation of Cryptococcus neoformans var. neoformans serotype D from eucalypts in South Brazil. Medical Mycology, 44(8):707-713.

Rondelli, M. C. H., Nogueira, A. F. S., Anai, L. A., Gava, F. N., Borin, S., Ondani, A. C., . . Santana, A. E. (2010). Criptococose diagnosticada por imprints de lesões em mucosa oral em cão. Arquivo Brasileiro de Medicina Veterinária e Zootecnia, 62(5):1271-1274.

Souza, V. L. d., Brandão, C. V. S., Minto, B. W., Estanislau, C. d. A., Ranzani, J. J. T., Babicsak, V. R., . . Ribeiro, M. G. (2014). Pleurisy associated with cryptococcosis in a dog: case report. Arquivo Brasileiro de Medicina Veterinária e Zootecnia, 66(5):1339-1342. 
Sykes, J. E., Hodge, G., Singapuri, A., Yang, M. L., Gelli, A. \& Thompson, G. R. (2017). In vivo development of fluconazole resistance in serial Cryptococcus gattii isolates from a cat. Medical Mycology, 55(4):396-401.

Trindade, D. B. M., Cavalheiro, A. L. B., Lopes, A. A., Santurio, S. T., Mazzanti, J. M. \& Eduardo Schossler, J. E. (2008). Diagnóstico de criptococose canina pela citologia aspirativa por agulha fina. Ciência Rural, 38(3):1-6.

Recebido: 29 de agosto, 2019.

Aprovado: 15 de outubro, 2019.

Publicado: 28 de novembro, 2019.

Licenciamento: Este artigo é publicado na modalidade Acesso Aberto sob a licença Creative Commons Atribuição 4.0 (CC-BY 4.0), a qual permite uso irrestrito, distribuição, reprodução em qualquer meio, desde que o autor e a fonte sejam devidamente creditados. 